\title{
Teoría y práctica: cuestiones imprescindibles a la práctica educativa
}

\author{
Cristiano Mezzarobai \\ Universidad Federal de Sergipe, Aracaju, SE, Brasil \\ Nicolás Carriquiriborde ${ }^{\mathrm{i} i(\mathbb{D})}$ \\ Universidad Nacional de La Plata, La Plata, Argentina
}

\begin{abstract}
Resumen
El texto, caracterizado como un punto de vista, se propone reflexionar en torno a la relación teoría y práctica en el contexto educativo, considerando la posibilidad de reflexión sobre una "teoría de la práctica educativa", de acuerdo con el autor argentino Ricardo Crisorio. Según el autor, una teoría de la práctica educativa debe elaborarse en el campo de la educación y debe permitirse ser útil sin ser compleja. En su metodología, se utilizó la perspectiva analítica interpretativa para discutir las ideas de Crisorio en la interfaz con los pensamientos de los más variados autores en el campo de la formación docente. Por lo tanto, se organizó el análisis, en un primer momento, presentando algunas notas conceptuales que delimitan la discusión, para luego explorar comprensiones, reflexiones y preguntas en la relación entre teoría y práctica. Teniendo en cuenta la dimensión del tiempo (reversible para la teoría e irreversible para la práctica) y pensando que estos dos polos - teoría y práctica - mantienen aspectos relacionados con la actividad humana que abarcan el pensamiento y la acción, estos son otros elementos reflejados en el texto.
\end{abstract}

\section{Palabras clave}

Teoría. Práctica. Práctica educativa. Ricardo Crisorio.

\section{Teoria e prática: questões imprescindíveis à prática educativa}

\section{Resumo}

O texto, caracterizado como um ponto de vista, propõe-se a refletir quanto à relação teoria e prática no contexto educacional, pensando na possibilidade de reflexão quanto a uma "teoria da prática educativa", de acordo com o autor argentino Ricardo Crisorio. Conforme este mesmo autor, uma teoria da prática educativa deve ser elaborada no campo da educação e deve permitir-se ser útil sem ser complexa. Metodologicamente se recorreu à perspectiva analítica interpretativa para discutir as ideias de Crisorio na interface com o pensamento dos mais variados autores do campo da formação de professores. Assim, organizaram-se as análises, em que, num primeiro momento, foram apresentadas questões conceituais que demarcam a discussão, para, na sequência, ser exploradas compreensões, reflexões e questões na relação entre teoria e prática. Considerando a dimensão do tempo (reversível para a teoria e irreversível para a prática) e pensando que esses dois polos - teoria e prática - guardam aspectos relacionados à atividade humana que englobam o pensamento e a ação, são outros elementos refletidos no texto.
\end{abstract}

\section{Palavras-chave}

Teoria. Prática. Prática educativa. Ricardo Crisorio. 
Theory and practice: essential questions for educational practice

\begin{abstract}
The text, characterized as a point of view, proposes to reflect on the relationship between theory and practice in the educational context, thinking about the possibility of reflection regarding a "theory of educational practice", according to the Argentine author Ricardo Crisorio. According to this author, a theory of educational practice must be developed in the field of Education and must be useful without being complex. Methodologically, we used the interpretive analytical perspective to discuss Crisorio's ideas at the interface with the thoughts of the most varied authors in the field of teacher education.Thus, we organize the analyzes in which, at first, we present conceptual questions that demarcate the discussion, in order to, next, explore understandings, reflections and questions in the relationship between theory and practice. To consider the dimension of time (reversible for theory and irreversible for practice) and think that these two poles - theory and practice encompass aspects related to human activity that englobe thought, action and performance are other elements reflected in the text.
\end{abstract}

\title{
Keywords
}

Theory. Practice. Educational practice. Ricardo Crisorio.

\section{Consideraciones iniciales}

Decimos que los alumnos saben cuando ellos consiguen reproducir exactamente lo que nosotros decimos: ¿eso es una buena práctica educativa? (CRISORIO, 2014).

En este texto, caracterizado como un punto de vista, reflexionamos en torno a la problemática siempre presente que envuelve la relación teoría y práctica, suscitada a partir de la provocación de lo que sería una teoría de la práctica educativa. Veamos: en la propia formulación de aquello que proponemos problematizar en este ejercicio reflexivo, los términos "teoría" y "práctica" están ahí, presentes, no opuestos, juntos. Sin embargo, cuando se establece esa reflexión sobre teoría y práctica, tan presente en el campo educativo, hay un sentido común entre los propios agentes del campo que procuran contraponer un término al otro en general con la frase: "En la práctica, la teoría es otra", o todavía: "Hablar y teorizar es muy fácil, te quiero ver en la práctica".

Partimos de la suposición de que, para que haya una práctica educativa, no hay forma de pensar acerca de tal acción, excepto en una articulación constante entre teoría 
y práctica, términos que están constantemente situados en polos separados, incluso de manera opuesta. Algunos dicen que la teoría y la práctica son paralelas que nunca se cruzan, es decir, caminan en paralelo, pero siempre separadas, sin diálogo, sin conexiones. Sería interesante pensar, desde nuestro punto de vista situacional -0 , como diría Bourdieu (2001), desde nuestras posiciones en el campo, como agentes -, que no hay forma de escribir este texto sino como una práctica basada en reflexiones teóricas.

Entonces, ¿por qué separar uno del otro? ¿Por qué la dificultad de articularlos? Si la educación superior es tan altamente valorada, ¿por qué lo que la caracteriza, que sería una capacitación teórico-conceptual como algo científico presentado y aprendido por académicos, se descuida cada vez más principalmente en los cursos de capacitación docente, en los cursos de Pedagogía y diferentes grados? Notamos que hay una crítica constante hacia los cursos de Pedagogía, por ejemplo, en el sentido de que son cursos con una carga excesivamente teórica (en Brasil, por ejemplo).

Por lo tanto, al formular este punto de vista, inicialmente tratamos de traer los significados, provenientes de los diccionarios (gramatical, filosófico, teórico, educacional, entre otros), de algunos términos, como "teoría" y "práctica" y tambien su sentido común. A partir de esto, utilizando algunos autores del campo de las ciencias humanas y sociales, como Adorno (2008), con algunos de sus aforismos en Minima moralia, y Rancière (2013), con sus lecciones sobre emancipación intelectual, entre otros (incluida la Educación Física, el campo de conocimiento al que estamos vinculados), exploraremos el tema ejercitando posibles formas de pensar sobre una articulación coherente entre teoría y práctica en el campo educativo, especialmente en el campo de la Educación Física escolar.

Elaborar esta reflexión ${ }^{1}$, entonces, se convierte en un ejercicio necesario e indispensable para seguir pensando en una cuestión intrínseca a las prácticas educativas, desde la formación humana hasta la formación profesional, que es la relación teoría-práctica. ¿Debemos preguntarnos, al colocarnos en el campo educativo,

1 Las reflexiones presentadas aquí son el resultado inicialmente de la participación de uno de los autores en un seminario especial realizado en el primer semestre de 2014 en el Programa de Posgrado en Educación de la Universidad Federal de Santa Catarina (UFSC), titulado "Por una teoría de la práctica educativa", cuyos profesores fueron el profesor doctor Ricardo Crisorio (La Plata/Argentina) y el profesor doctor Alexandre Fernández Vaz (UFSC). Posteriormente obtuvieron cuerpo del enfoque de los dos autores, cuando Nicolás Carriquiriborde, entonces estudiante de maestría de la Universidad Nacional de La Plata (UNLP), realizó un intercambio académico en el Programa de Posgrado en Educación de la Universidad Federal de Sergipe (UFS), de octubre a diciembre de 2019.

Educação \& Formação, Fortaleza, v. 5, n. 3, e2807, set./dez. 2020

DOI: https://doi.org/10.25053/redufor.v5i15set/dez.2807

https://revistas.uece.br/index.php/redufor/index 
qué hacemos con los otros? No podemos tener la falsa idea de que el objeto de la educación es el estudiante, es decir, el sujeto no puede ser el objeto. El objeto es el conocimiento y este conocimiento, en su construcción, transmisión, transformación, siempre se produce, transmite, transforma en una relación constante teórico-práctica entre sujetos o agentes.

Antes de pasar a cuestiones conceptuales, es importante presentar dos supuestos principales para que podamos pensar en una teoría de la práctica educativa, como lo señala Crisorio (2014): 1. Debe desarrollarse en el campo de la educación; 2. Debe servir, debe ser útil, de manera que alguien pueda servirse de ella.

Además de estos dos supuestos, es necesario, cada vez que estamos tratando con la dimensión de lo humano, considerar la diversidad humana, asumiendo todas las contradicciones que implican en el ser humano. Entonces, nos preguntamos: ¿Cómo producir una teoría en el campo educativo que nos permita abarcar diferentes hechos? Nos parece que, al entrar en la discusión, más que respuestas, vamos encontrando problemas para preguntar y discutir; aparecen dudas que complejizan la tentativa por abarcar y conceptualizar esta relación.

Giddens (2005, p. 528), por ejemplo, cuando trata del campo sociológico, especialmente el pensamiento teórico en Sociología, considera que la variedad teórica del campo, que podría ser un problema y un punto débil en Sociología, se caracteriza justamente por lo contrario: "[...] la variedad teórica nos libera del dogma". Tal sociólogo continúa, en relación con la dimensión humana, considerando que:

El comportamiento humano es complejo y multifacético y es muy poco probable que una sola visión teórica pueda cubrir todos los aspectos. La diversidad en el pensamiento teórico proporciona una rica fuente de ideas que pueden usarse en la investigación, además de estimular las capacidades imaginativas tan esenciales para el progreso del trabajo sociológico. (GIDDENS, 2005, p. 528).

Adorno (2008), en Minima moralia, libro repleto de aforismos, trae reflexiones a partir de la vida dañada. Para él, la vida de la sociedad está herida, dañada. En algunos de sus aforismos (una sentencia breve, una máxima), el autor se refiere a la cuestión teoría y práctica, principalmente debido al descuido que la sociedad viene teniendo en relación a la teoría. En el aforismo "Niño con agua de baño", Adorno (2008, p. 40) escribe que: 
Desde que se expulsó la utopía y se exige la unidad de teoría y práctica, existe un exceso de práctica. El miedo a la impotencia de la teoría ofrece la oportunidad de vender el alma al proceso de producción todopoderoso y, por lo tanto, reconocer la impotencia de la teoría de una vez por todas.

Un poco más adelante, se deduce: "Los seres humanos que se relacionan no deben guardar silencio sobre sus intereses materiales ni nivelarlos, sino que deben incorporarlos reflexivamente en su relación y con eso ultrapasarlos" (ADORNO, 2008, p. 41). Podemos desprender de eso una fuerte crítica de Adorno a la pérdida del peso que la teoría tiene sobre la práctica. Esto se manifiesta en nuestro mundo moderno por el pragmatismo de ser práctico, útil, objetivo, rápido, que termina sin producir confrontaciones, lo que también se presenta como algo incorrecto, teniendo en consideración que una teoría de la práctica tiene su utilidad y legitimidad.

Nos parece que hoy se está pensando en algo solo para dar cuenta de cuestiones prácticas. En el campo de la formación profesional, especialmente en Pedagogía y profesorados (y quizás en otros también), la caracterización curricular está configurada por la formación espejo ${ }^{2}$, que demuestra, en cierto modo, la crítica de vanguardia de Adorno con respecto al desprecio de los temas teóricos en la formación humana: al futuro profesor cabe pasar mucho tiempo en la escuela, observando las prácticas de maestros que ya trabajan en ese contexto, en lugar de ser presentados (y aprender/aprehender) teorías, conceptos, proposiciones didáctico-pedagógicas y debates epistemológicos de la formación humana, social y cultural.

Otro aforismo que podemos aportar a nuestro ejercicio reflexivo se titula "Para Marcel Proust", en el cual Adorno (2008) comenta que incluso el trabajo intelectual no puede estar libre de las aporías del mundo administrado. Los intelectuales, defiende Adorno (2008), necesitan independencia y autonomía ${ }^{3}$. La cuestión de los intelectuales es

2 La "noción del espejo", cuando se piensa en la formación del profesorado, sería la forma en que se intenta abordar la práctica como algo que promueve el aprendizaje de los futuros docentes, aunque se sabe que tal experiencia no da cuenta de una formación realmente completa, integral y crítica (MEZZAROBA, 2016).

3 ¿Qué diría Adorno si estuviese vivo al saber del movimiento que en Brasil se conoce como "Escola sem Partido"? Se trata de un movimiento que desde 2004, aunque sin tantos enfoques, fue iniciado por el fiscal del estado de São Paulo Miguel Nagib, quien creó, coordinó y difundió el movimiento que se estaba expandiendo bajo el pretexto de varias "banderas" (ideológicas, religiosas, mercadológicas, etc.). Luego, la Cámara de Diputados comenzó a discutir, a través del Proyecto de Ley n 867/2015 (del diputado Flavio Augusto da Silva, del Partido Social Cristiano de São Paulo), que sería una forma de censura para los profesores brasileños y limitaría su propia autonomía y libertad de pensamiento al actuar en la educación de jóvenes y adultos, propagando un discurso de "neutralidad ideológica" frente al "adoctrinamiento" que ciertos maestros hacen en sus clases (tipificando el "acoso ideológico" en el

Educação \& Formação, Fortaleza, v. 5, n. 3, e2807, set./dez. 2020

DOI: https://doi.org/10.25053/redufor.v5i15set/dez.2807

https://revistas.uece.br/index.php/redufor/index 
algo que Adorno (2008, p. 18) siempre aborda: "[...] algunos [...] necesitan adaptarse para vivir, y aquellos que podrían vivir sin él son rechazados porque no quieren adaptarse".

En el aforismo "Cuando malvados te atraen", Adorno (2008) no ve ninguna posibilidad de un grupo de resistencia en la sociedad administrada. Regresa a la pregunta/papel de los intelectuales, pensando que hay una conformidad para esta clase: "[...] crece de tal modo el esfuerzo para producir algo que de algún modo se sustente que casi nadie consigue llevarlo a cabo. Además, la presión de conformidad, que pesa sobre cada productor, deprime su propia demanda" (ADORNO, 2008, p. 25).

Desde este punto de vista, entonces, reflexionamos sobre la relación entre teoría y práctica, pensando en una teoría de la práctica educativa. En este primer momento, como vimos, trajimos una breve contextualización que marca la discusión y, a continuación, exploraremos las comprensiones, reflexiones y preguntas de esta relación.

\section{Teoría y práctica: comprensiones, reflexiones, preguntas}

Pasemos a las posibles comprensiones de los términos que estamos considerando centrales en nuestro ejercicio reflexivo. Comenzaremos, antes de eso, con el término "sentido común", por el carácter que este determina en aquellos términos-claves para nuestra reflexión. Para el Diccionario de Teoría y Educación Cultural (SILVA, 2000, p. 99), "sentido común" es un término que, a partir de los análisis y teorizaciones del filósofo italiano Antonio Gramsci, sería el "[...] conjunto de supuestos que son parte de las visiones de mundo poco organizadas, contradictorias y fragmentadas de cualquier sociedad. En un sentido amplio, el conjunto de presupuestos sociales que se aceptan sin cuestionamiento". Procuraremos alejarnos de tales presupuestos, ya que buscamos cuestionar y problematizar los términos centrales: primero teoría, después práctica.

aula como "crimen"). Con la destitución de la presidenta Dilma Rousseff en 2016 y las elecciones presidenciales en 2018, el movimiento se extendió y creció en controversia, generando movimientos de resistencia, principalmente de agentes que trabajan en escuelas y universidades, que afirmaron que no se trata de una "Escuela sin Partido", pero sí de una "Escuela sin Libertad". El año 2018 terminó y el referido proyecto no fue votado por la Comisión de la Cámara y fue temporalmente "archivado". Aunque no se ha convertido en ley, es innegable que la propuesta ha tenido un fuerte impacto en la educación brasileña, especialmente con respecto a la calidad de la educación, en cuanto a los dogmatismos, a la censura y a la persecución. Para obtener más información, consulte Polizel (2019).

Educação \& Formação, Fortaleza, v. 5, n. 3, e2807, set./dez. 2020

DOI: https://doi.org/10.25053/redufor.v5i15set/dez.2807

https://revistas.uece.br/index.php/redufor/index 
Según el Diccionario Aurélio (2004), en lengua portuguesa, la palabra "teoría" puede incluir varias denotaciones. Originaria del griego "theoría", sería "[...] acción de contemplar, examinar"; "estudio", y todavía:

1. Conhecimento especulativo, meramente racional. 2. Conjunto de princípios fundamentais duma arte ou duma ciência. 3. Doutrina ou sistema fundado nesses princípios. 4. Opiniões sistematizadas. 5. Noções gerais; generalidades: a teoria do cinema. 6. Suposição, hipótese. 7. Utopia; quimera. [...] 11. Filos. Conjunto de conhecimentos não ingênuos que apresentam graus diversos de sistematização e credibilidade, e que se propõem explicar, elucidar, interpretar ou unificar um dado domínio de fenômenos ou de acontecimentos que se oferecem à atividade prática. 12. Lóg. Do ponto de vista estritamente formal, o sistema de proposições em que não se encontram proposições contraditórias, nem nos axiomas, nem nos teoremas que deles se deduzem.

Percibimos, por tanto, variadas significaciones, desde aquellas que apuntan que puede ser comprendido como algo que disminuye la potencia del término, como el hecho de ser una utopía, una quimera ${ }^{4}$ o una mera suposición, como aquellas que delegan a la teoría una opinión más sistematizada, configurándose en un arte o una ciencia, basado en principios, permitiendo la creación de nociones generales. Ya en el Diccionario de la Lengua Española (Real Academia Española), el término "teoría” está así explicitado:

1. f. Conocimiento especulativo considerado con independencia de toda aplicación. $2 \mathrm{f}$. Serie de las leyes que sirven para relacionar determinado orden de fenómenos. $3 \mathrm{f}$. Hipótesis cuyas consecuencias se aplican a toda una ciencia o a parte muy importante de ella. $4 \mathrm{f}$. Entre los antigos griegos, procesión religiosa. Teoría de cuerdas, o teoría de supercuerdas. 1 f. Fís. teoría unificada del universo, en la que se afirma que los constituyentes básicos de la naturaleza no son partículas fundamentales, del tipo del eléctron, sino ultradiminutos filamentos unidimensionales llamados cuerdas. En teoría. 1 loc. adv. Sin haberlo comprobado en la práctica. (RAE, 2019).

Tenemos algunas similitudes en el Diccionario de Lengua Española con lo que aparece en el Diccionario de Lengua Portuguesa, principalmente cuando la teoría es colocada como un conocimiento especulativo y se constituye en una serie de principios ordenados que dan orden a los fenómenos y que se aplican a la ciencia o parte de ella. Sin embargo, en el diccionario citado en lengua portuguesa, no hay oposición con la dimensión de la práctica, diferentemente de lo que ocurre en la versión de la lengua española, cuando informa que "teoría" es algo que todavía no fue comprobado por la

4 O como un "discurso vacío", segun Marcellino (1995, p. 74). 
práctica, lo que nos lleva a interpretar la práctica siendo un criterio de legitimación de la teoría, jerarquizando ambas.

Cuando buscamos el significado de "teoría" en un Diccionario Básico de Filosofía (JAPIASSU; MARCONDES, 2001, p. 183), encontramos lo siguiente:

teoria (fr. théorie, do lat. e do gr. theoria). 1. Na acepção clássica da filosofia grega, conhecimento especulativo, abstrato, puro, que se afasta do mundo da experiência concreta, sensível. Saber puro. Sem preocupação prática. 2. Modelo explicativo de um fenômeno ou conjunto de fenômenos que pretende estabelecer a verdade sobre esses fenômenos. Determinar sua natureza. Conjunto de hipóteses sistematicamente organizadas que pretende, através de sua verificação, confirmação ou correção, explicar uma realidade determinada. Ex.: a teoria da relatividade de Einstein. Ver ciência; explicação; método.

Aquí, visualizamos una dicotomía entre lo que sería la experiencia del mundo inteligible versus la experiencia del mundo sensible. La teoría estaría vinculada al primer caso, siendo la razón y controladora de la experiencia sensible. Sería el conocimiento verdadero que alcanzamos exclusivamente por el pensamiento.

Ahora pasamos al término "práctica". El Diccionario Aurélio (2004) contiene lo siguiente en relación con la "práctica": "[Dev. de praticar.] Substantivo feminino. 1. Ato ou efeito de praticar. 2. Uso, experiência, exercício. 3. Rotina; hábito. 4. Saber provindo da experiência; técnica. 5. Aplicação da teoria. [...] 8. Filos. Práxis (2). [Cf. pratica, do v. praticar]". Percibimos, a primera vista, el problema relacionado con la aplicación, la ejercitación, algo que se realiza como hábito ${ }^{5}$, por medio de determinadas técnicas (incluso sencillas) que van siendo rutinizadas. También vemos más arriba una relación con teoría, es decir, la práctica sería la aplicación de una teoría. Cuando visualizamos el significado de "práctica" en el Diccionario de la Lengua Española (RAE, 2019), tenemos la siguiente descripción:

1. adj. Perteneciente o relativo a la práctica. 2. adj. Dicho de un conocimiento: Que enseña el modo de hacer algo. 3. adj. Experimentado, versado y diestro en algo. 4. adj. Que piensa o actúa ajustándose a la realidad y persiguiendo normalmente un fin útil. 5. adj. Que comporta utilidad o produce provecho material inmediato. 6. m. Técnico que, por el conocimiento del lugar en que navega, dirige el rumbo de las embarcaciones en la costa o en un puerto. 7. f. Ejercicio de cualquier arte o facultad, conforme a sus reglas. 8. f. Destreza adquirida con el ejercicio de la práctica. 9. f. Uso continuado, costumbre o estilo de algo. 10. f. Modo o método que particularmente observa alguien en sus operaciones. 11. f. Ejercicio que bajo la dirección de un maestro y por cierto tempo tienen que hacer algunos para habilitarse y poder ejercer públicamente su

5 Una tarea o acción, como regla, desprovista de sentido según Marcellino (1995).

Educação \& Formação, Fortaleza, v. 5, n. 3, e2807, set./dez. 2020

DOI: https://doi.org/10.25053/redufor.v5i15set/dez.2807

https://revistas.uece.br/index.php/redufor/index 
profesión. [...] 12. f. Aplicación de una idea o doctrina. 13. f. Contraste experimental de una teoría. En la práctica. 1. loc. adv. Casi en realidad. Llevar a la práctica. 1. loc. verb. poner en práctica. Poner en práctica. 1. loc. verb. Realizar ideas, planes, proyectos, etc.

Se explicita la idea de acción, de un hacer, una experimentación, una actuación, en vista de un fin práctico a partir de un conocimiento dado. $\mathrm{O}$, de igual modo, la idea de un ejercicio que ocurre por el hábito y acción continuada, la aplicación de una teoría (por medio de una técnica específica), bien como, conforme vimos, una diferencia colocada a la prueba entre la práctica en relación a la teoría.

Esa misma denominación, en el Diccionario de Filosofía Básica (JAPIASSU; MARCONDES, 2001, p. 155, nuestra traducción), es tratada de la siguiente manera:

práctica/práctico (gr. praktikós, de prattein: agir, realizar, fazer) Que concierne a la acción. Acción que el hombre ejerce sobre las cosas, aplicación de un conocimiento en una acción concreta, efectiva. Ej.: 'saber práctico'. Conocimiento empírico, saber hacer algo. Ej.: 'práctica pedagógica', 'práctica médica'. Opuesto a teórico, especulativo. 2. Razón práctica. Según Kant, respondemos a la cuestión teórica ‘¿qué podemos saber?'. Por el examen de las condiciones a priori del conocimiento; en tanto que respondemos a la cuestión práctica, '¿qué debemos hacer?', por el establecimiento de las leyes de la acción moral. 'Tudo na natureza age de acordo com leis. Há apenas um ser racional que tem a faculdade de agir a partir da representação das leis, isto é, a partir dos princípios, em outras palavras, que tem vontade. Uma vez que para derivar as ações das leis a razão é necessária, a vontade não é senão a razão prática' (Kant. Metafisica dos costumes).

En la propia definición filosófica del término, "práctica" se coloca como opuesto de "teoría". En el ejemplo, una práctica pedagógica sería opuesta al conocimiento teórico. Es interesante notar que, en el ejemplo arriba mencionado, dicha práctica se opondría al conocimiento teórico. Saber hacer algo y aplicar ciertas acciones no estaría relacionado a un conocimiento en el plano teórico. Presupone una acción de un sujeto sobre las cosas, como si la teoría no fuese una acción de sujetos sobre sus propias preguntas/cosas, una acción sobre el mundo.

Lo que está claro hasta aquí es que, denotativa y filosóficamente, los términos "teoría" y "práctica" son colocados como antagonistas, lo que también reforzaría, en parte, el sentido común de que ambos no dialogan, se excluyen y tampoco se complementan entre sí. Es notable que el sentido común determina la primacía de la práctica sobre la teoría, sobrevalorizando aspectos prácticos (de la acción) en relación a los aspectos teóricos (del conocimiento y el pensamiento más racional y reflexivo). 
Para Pinto (1995), existe la necesidad de pensar en un puente entre ellos, ya que ni toda teoría es enteramente traducida en la práctica, ni toda práctica es completamente teorizable. Según esta autora: "La teoría y la práctica son polos que tienen su sentido propio, pero, al mismo tiempo, mantienen entre sí una relación recíproca y simultánea de autonomía y dependencia de una en relación a la otra" (PINTO, 1995, p. 137, nuestra traducción).

Sin embargo, es necesario considerar una diferencia entre la teoría y la práctica: se sitúa en relación con el tiempo; teoría y práctica se relacionan de diferente manera. El tiempo de la teoría es reversible; el tiempo de la práctica no lo es (por ejemplo, al planificar la lección, podemos rehacerla; el tiempo de la práctica, el evento de la lección, es inexorable: tiempo lógico, tiempo cronológico) (CRISORIO, 2014).

De este modo, podríamos pensar que la práctica esta urgida por las acciones, es aquel momento donde predomina el tiempo de las acciones, de la toma de decisiones afectando la propia práctica. Incorporando esta dimensión, podemos afirmar que en la práctica no hay regreso posible. En cambio, la teoría es una práctica de otro orden, es más bien un tiempo de reflexión. Es un tiempo que se distancia de la acción, es un tiempo, si se quiere, reversible. De modo simple, podemos decir que en la práctica prevalece la acción práctica, no la reflexión. Del mismo modo, en la teoría prevalece la reflexión, no la acción.

Crisorio (2014), al interrogar la relación entre teoría y práctica, se pregunta: ¿tal oposición o relación tensa entre ambos sería solo una cuestión de lenguaje, de construcción discursiva? En varios ámbitos de la vida cotidiana, especialmente en el mundo académico, nos enfrentamos a dicotomías ${ }^{6}$ : naturaleza $x$ cultura; sujeto $x$ alma; pensamiento $\mathrm{x}$ acción, teoría $\mathrm{x}$ práctica. No podemos pensar que hay algo completamente puro. Las teorías no son más que productos del pensamiento y lenguaje humanos. Por lo tanto, probablemente cualquier intento de transformar la oposición explícita entre teoría y práctica estaría en el plano posible de las palabras, más que de las acciones.

6 Para Marcellino (1995, p. 74), "dicotomía" es la "[...] división de un concepto en otros dos, en general contrarios, que agotan su extensión”. En sus reflexiones, aborda la dicotomía de la relación teoría/práctica, en que, según este autor, "[...] vienen cargados con una división, de una cisura, que se presenta como antagonista, muchas veces, y que no debería serlo, por el propio significado de cada uno de ellos. Además, deberían ser entendidos, englobados, en un único concepto, que no agote su extensión y no los coloque en campos contradictorios". 
Si tomamos la interpretación foucaultiana sobre prácticas como "[...] sistemas de acción en la medida en que están habitados por el pensamiento" (CASTRO, 2011, p. 316), podemos afirmar que, en cada acción, en la práctica, existe una serie de reglas, de conjuntos prácticos (FOUCAULT, 1991) que organizan esos modos de obrar, esas acciones. Desde esta perspectiva, podemos hacer una distinción entre prácticas y acciones, donde estas se encuentran habitadas por un conjunto de enunciados que le dan sentido y son los modos de obrar que los sujetos poseen en un tipo particular de sistema práctico, de modo que la acción no antecede a la práctica, ni mucho menos puede separársela de ella, más bien la acción procede y es efecto de la práctica. En este sentido, toda posibilidad de percepción, pensamiento y acción depende de la estructuración de un cierto campo significante que preexiste a lo factual (LACLAU, 1996).

Entonces ya no solo la acción, sino que los modos de pensar, decir y hacer se encuentran habitados por prácticas, que, a la manera foucaultiana, son prácticas discursivas. Aquí la noción de discurso se refiere no a un conjunto de signos que designarían una serie de cosas, es decir, una suerte de representación de cosas que están allí antes o más allá del discurso, sino "[...] como el acto de significación que es terreno primario de la objetividad" (BUENFIL BURGOS, 2008, p. 118).

Desde esta perspectiva, el discurso antecede a la acción y al pensamiento en tanto que "[...] remite a la totalidad significativa productora de sentido que estructura la vida social y de la que los sujetos no son plenamente conscientes" (SOUTHWELL, 2017, p. 121). Así, teoría y pensamiento se ven también afectados por prácticas discursivas en la medida que las entendemos como actividades propiamente humanas, por tanto, afectadas por un orden significante que les otorga sentido.

Coincidimos con Crisorio (2018, p. 7) que la práctica:

[...] no supone un sujeto practicante, que precede a las prácticas y las realiza; antes bien, indica un sujeto 'practicado', precedido por las prácticas y realizado en ellas. Del mismo modo, la idea de pensamiento no implica un sujeto que lo piensa, preexistiéndolo, sino más bien un sujeto 'pensado', constituido en y por el pensamiento.

Delimitada la noción de práctica y en articulación a ella, podemos conceptualizar los términos "teoría", "pensamiento" y "acción", pero ya no desde algún fundamento trascendental que contenga lo esencial de cada concepto, sino más bien pensarlos como un tipo de actividad, es decir, no por su contenido como sustantivo, sino como verbo. 
Entonces, podemos vincular el pensamiento a la actividad de pensar. En ella predomina un tipo de actividad que busca reflexionar sobre algún aspecto, saber u objeto. Al pensar predomina la actividad reflexiva. Puede ser o no sistemático, comunicable, verificable, sin embargo, no deja de ser una actividad del pensamiento, de la abstracción, y que puede prescindir de la materialización en algo observable, palpable.

Teorizar refiere también a una actividad del pensamiento, pero de otro grado. Teorizar se vincula inevitablemente con el conocimiento, el saber, sin embargo, a diferencia del pensamiento, debe guardar cierto grado de sistematicidad, comunicabilidad y método. Teorizar es una actividad reflexiva, sistemática, metódica, comunicable sobre algún aspecto cualquiera de la cultura.

En cambio, la acción supone actuar, mover algo, afectar el curso de algo. No es una abstracción, supone un movimiento. Si accionamos, afectamos algo. La variable tiempo es irreversible, no hay posibilidad de volver atrás lo que se afectó. Cabe aclarar que no estamos afirmando que un pensamiento y, más aún, una teoría no afecten el curso de lo dado. Solo a los efectos de nuestra argumentación nos proponemos pensarlos en su condición verbal y no sustancial. Así podemos comenzar a pensar los términos "teoría" y "práctica", pensamiento y acción, como estrechamente vinculados, sin confundirlos, en su distinción. La práctica, en el sentido que venimos desarrollando, es el terreno discursivo que produce una totalidad significativa y en el que los modos de pensar, decir y hacer se ven afectados. Con esto, no hay actividad alguna que quede por fuera de la práctica (discursiva). Entonces nuestras formas de pensar y actuar siempre estarán afectadas por la práctica discursiva.

Sin embargo, eso no nos puede hacer confundir la actividad del pensar con la actividad del accionar. En una prevalece la actividad de la reflexión, de la abstracción: puedo reflexionar sobre la acción que lleve adelante en una clase, un deporte o el trabajo. Estoy fuera del tiempo de la acción, estoy en un tiempo reversible, analizo mi intervención en la clase, mis decisiones en un deporte, mi desempeño en el trabajo. Prevalece la actividad del pensar.

En la otra prevalece la acción. Si acciono, estoy afectando el curso de lo dado, muevo algo, lo hice, accioné, no hay vuelta atrás. El deportista pasó, remató, se alejó. El docente intervino de una manera en la clase. El artista actuó, cantó, tocó. No hay ausencia del pensamiento, sin embargo es el tiempo de la acción. 
Si nos ceñimos a estas dimensiones de cada una, podemos reubicar la discusión teoría-práctica de un modo - entendemos - más sencillo (lo que no quiere decir que se acabe la discusión). Existe un tiempo para la acción y un tiempo para la reflexión, para el pensar. Esto nos exime, por otro lado, de suponer que existe un saber propio de la acción (o lo que se entiende en el sentido común de la práctica). El saber surge de la actividad reflexiva que ejerzo sobre lo dado, accionado. No está en lo dado ni en las acciones, está en la reflexión. Cuando una reflexión se vuelve un campo de conocimiento sistemático, comunicable, verificable y metódico, hablamos de teoría. Recapitulando, con Crisorio (2014) podemos pensar tal relación como una teoría de la práctica educativa, considerando que la teoría no resuelve los problemas de una práctica. La teoría, precediendo la práctica, ayuda a pensarla. Según el autor, la teoría sirve para pensar la práctica y ayuda en la toma de decisiones.

Vemos, por lo tanto, la necesidad de escapar de las relaciones jerárquicas entre uno y otro polo, es decir, la teoría no debe verse como algo utilitario para la práctica, pero sí articulada a esta última, una dialogando con la otra, una mostrando caminos posibles a la otra, en un diálogo constante y con tensión para superar los problemas pedagógicos (a veces van más allá del alcance pedagógico) y orientar posibilidades a las problematizaciones que ocurren en el cotidiano trabajo de enseñar y en la propia cultura escolar.

Aún para Crisorio (2014), siguiendo sus notas sobre el tema planteado, una teoría satisfactoria precisa:

- ser simple, tener pocos elementos, lo que garantizaría la potencia (en otras palabras, el foco es en la objetividad, en la concentración en el objeto; pero también podríamos pensar: ¿simplificar para complejizar?);

- ser elegante: es decir, que los pocos elementos que el modelo (la teoría) contiene puedan explicar y predecir de manera sencilla sus resultados.

Quizás, si pensamos en formas de enseñar, y ahí la articulación de la teoría y la práctica, deberíamos volver a la idea de la problematización. Si la educación es introducir al joven en la cultura para que sea protagonista, partícipe y crítico de su mundo, entonces el ejercicio que permitirá la combinación adecuada de teoría y práctica debería ser a través de la problematización: ¿las acciones fueron así? ¿Son así? ¿Siempre serán así? 
¿Deberían haber sido siempre así? ¿Cómo serán si no es como son ahora? Todo eso es problematizar, cuestionar lo que es dominante (CRISORIO, 2014).

Esto nos remite a lo que Rancière (2013, p. 44) sugiere con respecto a la forma en que el alumno o alumna debe ser tratado en el contexto escolar: "El alumno debe ver todo por sí mismo, comparar incesantemente y responder siempre la triple pregunta: ¿qué ves?, ¿qué pensas de eso?, ¿qué haces con eso? Y así, hasta el infinito". Rancière (2013, p. 51) aún, a nuestra manera de ver una sugerencia de cómo lidiar con el ejercicio de problematización por parte del maestro, indica que: "Siempre hay algo que el maestro le puede pedir [al alumno] que descubra, sobre el que puede interrogarlo y verificar el trabajo de su inteligencia".

Pensando en el ámbito de la relación profesor-alumno y en el trabajo teóricopráctico del maestro en relación con aquellos bajo su responsabilidad pedagógica, Rancière (2013, p. 56-57) nos ofrece algunas pistas sobre lo que puede ser una práctica educativa más eficiente:

Así es como el maestro ignorante puede instruir tanto al que sabe como al ignorante: verificando si él está investigando continuamente. Quien busca, siempre encuentra. No encuentra necesariamente aquello que buscaba, menos todavía aquello que es preciso encontrar. Pero encuentra alguna cosa nueva para relacionar lo que ya conoce. Lo esencial es esta vigilancia continua, esa atención que nunca se relaja sin que se instale la sinrazón - en la que exceden tanto al que sabe como al ignorante. El maestro es el que mantiene lo que busca en su camino, donde está solo para buscar y procura hacerlo incesantemente.

La búsqueda constante de la excelencia (no estamos hablando de perfección, que históricamente ha guiado los cursos de mejoramiento de los docentes) en relación con la formación de docentes requiere que la relación entre la teoría y la práctica se trate de manera equilibrada, que una no se superponga a la otra, para que, en el ejercicio de las prácticas educativas, el conocimiento se aborde y trabaje en su amplitud, complejidad y profundidad, permitiendo que fenómenos contingentes, tan presentes en el ámbito educativo, no sean desconsiderados porque la teoría no los anticipó, pero si porque son propios de la práctica.

Contreras (2012, p. 288), haciendo referencia a la cuestión de la práctica educativa, principalmente en la forma como las políticas públicas desestabilizan y desmovilizan la dimensión de la formación humana por la vía de la autonomía escolar, llama la atención al hecho de que la escuela no se torne "[...] uma instituição voltada para 
si mesma, como uma empresa que deve definir seu produto [...]". Según este autor, eso implicaría una enseñanza que pierde su "[...] sentido político (que significaria a consciência da educação como prática que tem pressupostos e consequências sociais, devendo ser objeto de participação, deliberação e acordo social), ficando transformado em uma questão meramente técnica" (CONTRERAS, 2012, p. 288).

Entonces, no podemos perder de vista que, en la discusión presente, en cuanto al par teoría-práctica, la dimensión política debe ser colocada como aspecto relevante, porque de nada sirve una teoría que no se implique en el contexto educativo y pedagógico, de la misma manera que de nada sirve una práctica (acciones, técnicas, haceres) que se supone vacía de significados a la vida en sociedad de los seres aprendentes.

Sobre eso, Giroux (1997, p. 29, nuestro énfasis) hace la siguiente contribución y también nos ayuda a pensar la práctica educativa contextualizada y politizada:

\begin{abstract}
[...] Ao politizar-se a noção de escolarização, torna-se possível elucidar o papel que educadores e pesquisadores educacionais desempenham enquanto intelectuais que operam em condições especiais de trabalho e que desempenham uma função social e política particular. As condições materiais sob as quais os professores trabalham constituem a base para delimitarem ou fortalecerem suas práticas como intelectuais. Portanto, os professores enquanto intelectuais precisarão reconsiderar e, possivelmente, transformar a natureza fundamental das condições em que trabalham. Isto é, os professores devem ser capazes de moldar os modos nos quais o tempo, espaço, atividade e conhecimento organizam o cotidiano nas escolas. [...] Em última análise, os professores precisam desenvolver um discurso e conjunto de suposições que Ihes permita atuarem mais especificamente como intelectuais transformadores. Enquanto intelectuais, combinarão reflexão e ação no interesse de fortalecerem os estudantes com as habilidades e conhecimentos necessários para abordarem as injustiças e de serem atuantes críticos comprometidos com o desenvolvimento de um mundo livre da opressão e exploração.
\end{abstract}

Considerando la producción existente en el ámbito de la educación, en especial las discusiones que envuelven teoría y práctica, sabemos que el paradigma de la racionalidad técnica, que tiene como principio una solución instrumental de los problemas, no da cuenta de la complejidad que envuelve los contextos diversos de las prácticas educativas. Giroux (1997, p. 25) recuerda que: "[...] en la visión del mundo de los tradicionalistas, las escuelas son simplemente un lugar de instrucción. Se ignora que las escuelas son también lugares políticos y culturales [...]”. 
A partir de una mirada epistemológica de las prácticas, tenemos la contribuición de autores como Charlot $(2005)^{7}$ y Tardif $(2000)^{8}$, quienes investigan los saberes docentes; así como de Schön $(1992)^{9}$, que tratará en cuanto al profesor reflexivo, o mismo Stenhouse $(1975,1991)^{10}$ en relación al profesor investigador. Además de ellos, también tenemos las producciones em cuanto al intelectual crítico envolviendo Henry Giroux, Paulo Freire, Peter McLaren y también Antonio Gramsci: autores que piensan y problematizan el intelectual como categoría central que permite articular las dimensiones políticas y pedagógicas, cuyo objetivo principal es la emancipación humana.

\section{Consideraciones finales}

\section{Entendemos que existe una búsqueda incesante en el campo educativo para} comprender mejor y apropiarse mejor de esta aventura, que es pensar la relación entre teoría y práctica, en la vigilancia de no caer en el sentido común. Lo que se muestra, incluso con los avances en el campo científico y en el contexto educativo, es que resulta un gran enemigo de las prácticas pedagógicas educativas de los y las docentes.

Terminando nuestro ejercicio reflexivo, en el contexto de esta tensa relación entre la teoría y la práctica, buscamos, sin caer en las oposiciones vigentes o en la forma actual de sobrevalorizar los aspectos prácticos de la formación en detrimento de los

7 Para Charlot (2005, p. 90), "Formar alguém é torná-lo capaz de executar práticas pertinentes a uma dada situação, definida de maneira restrita (função no trabalho) ou ampla (em referência a um setor de trabalho encarregado de um processo de produção)". Para él, formar profesores sería el trabajo con saberes y prácticas o, en sus palabras, la práctica del saber y los saberes de la práctica, esa constante tensión - o mediación - entre lo abstracto y lo concreto.

8 Tardif (2000) afirma que los cursos de formación no han dado cuenta de una formación profesional adecuada, por estar centrados en el saber científico, proponiendo que los saberes producidos por los propios profesores seam utilizados en el cotidiano escolar. Más allá de eso, el autor hace una clasificación de los saberes profesionales de los profesores (temporales, plurales, heterogéneos, personalizados, situados, con marcas del ser humano) y defiende abandonar la lógica disciplinar en favor de una lógica profesional centrada en el estudio de los trabajos reales del profesor.

9 Conforme Schön (1992), tenemos cómo comprender las actividades espontáneas del cotidiano a partir del conocimiento en la acción, que es el conocimiento tácito, espontáneo y no sistematizado; y por la reflexión en la acción, o sea, la reflexión realizada para analizar algo frente a una situación en que nos encontramos. Para el referido autor, es el proceso de reflexión en la acción lo que transforma al profesional en un investigador en el contexto de la práctica, entendiendo que el profesor no depende de técnicas preestablecidas, él crea una nueva manera de ver el problema.

10 Stenhouse (1991) propone que el profesor debe tener autonomía en relación a la selección y al trato de los contenidos escolares, valorizando la investigación sobre la práctica educativa, o sea, defiende que cabe al profesor pensar y producir conocimiento a partir de la experiencia práctica de la investigación en la clase.

Educação \& Formação, Fortaleza, v. 5, n. 3, e2807, set./dez. 2020

DOI: https://doi.org/10.25053/redufor.v5i15set/dez.2807

https://revistas.uece.br/index.php/redufor/index 
aspectos teóricos, visualizar aspectos de crítica y creatividad con respecto a las prácticas educativas. Con esto, queremos decir que tomar posesión de la dimensión teórica, hacer contacto con la realidad de las prácticas educativas permitirá a los sujetos en el campo educativo pensar en teorías de sus propias prácticas educativas, ya que, como sabemos, nos referimos a lo humano y especialmente a su educación/ capacitación. No hay formas mágicas ni recetas de cocina que estandaricen comportamientos, acciones y objetivos únicos. Por lo tanto, considerar los aspectos singulares de cada contexto educativo, formativo, social y cultural permitirá a cada agente en este campo ejercer su actividad humana movilizando elementos reflexivos y acciones prácticas contextualizadas.

Si entendemos las prácticas educativas como acciones pensadas, reflejadas y experimentadas por los docentes como intelectuales transformadores, entonces sabemos la indispensabilidad de combinar la reflexión y la acción, o sea, teoría y práctica, con una mirada a una educación que problematice la realidad, opere en el presente y revele mecanismos de injusticia, dominación y opresión, siempre recurrentes e insistentes.

Así, cuando volvemos al epígrafe de este texto, en el que Crisorio (2014) pregunta: "Decimos que los alumnos saben cuando ellos consiguen reproducir exactamente lo que nosotros decimos: ¿eso es una buena práctica educativa?", se queda evidente que en la educación actual la reprodución sin reflexión y como puro automatismo ya no puede considerarse una práctica educativa coherente con los individuos que hoy participan en el campo educativo y formativo.

Una teoría de la práctica educativa debe ser formulada por cada sujeto que actúa en su contexto, a partir de las diversas teorías existentes (que podrán ser testadas, pero, antes de eso, deben ser presentadas, aprehendidas, reflexionadas, cuestionadas, tensionadas) y accionadas en su contexto específico. Recordando, como sostiene Crisorio (2014), una teoría de la práctica educativa debe desarrollarse dentro del campo de la educación y debe ser útil para sus agentes y participantes. Pero atención: no nos estamos refiriendo a un cierto eclecticismo, algo que está muy de moda hoy en día (que anula el conocimiento epistemológico básico), sino más bien a la comprensión de la cultura mosaico que son los campos de formación, ya que cada campo profesional elige sus teorías, metodologías y conocimientos generales y específicos. 
Es necesario que se nos presente todos esos conocimientos (teóricos, de procedimiento, metodológicos, prácticos y técnicos) para tomar posición en el campo del que formamos parte: ique sepamos hablar y argumentar pedagógicamente, con propiedades teóricas y prácticas, sobre aquello que hacemos en nuestro día a día!

\section{Referencias}

ADORNO, T. W. Minima moralia: reflexões a partir da vida lesada. Rio de Janeiro: Beco do Azougue, 2008.

BOURDIEU, P. Para uma sociologia da ciência. Lisboa: 70, 2001.

BUENFIL BURGOS, R. N. (Org.). Giros teóricos de las ciencias sociales y las humanidades. Córdoba: Comunicarte, 2008.

CASTRO, E. Diccionario Foucault: temas, conceptos y autores. Buenos Aires: Siglo XXI, 2011.

CHARLOT, B. Ensinar, formar: lógica dos discursos constituídos e lógica das práticas. In: CHARLOT, B. Relação com o saber, formação de professores e globalização: questões para a educação de hoje. Porto Alegre: Artes Médicas, 2005. p. 89-99.

CONTRERAS, J. A autonomia de professores. 2. ed. São Paulo: Cortez, 2012.

CRISORIO, R. Prácticas corporales en educación corporal. In: COLOQUIO ESTUDIOS SOBRE LA EDUCACIÓN DEL CUERPO, 1., 2018, Montevideo. Ponencia... Montevideo: ISEF-UdelaR, 2018.

CRISORIO, R. Seminário especial: por uma teoria da prática educativa. Exposições no Seminário Especial realizado no Programa de Pós-Graduação em Educação da UFSC. Florianópolis: UFSC, 2014.

FOUCAULT, M. ¿Qué es la ilustración?. Madrid: De la Piqueta, 1991.

GIDDENS, A. Sociologia. 4. ed. Porto Alegre: Artmed, 2005.

GIROUX, H. A. Os professores como intelectuais: rumo a uma pedagogia crítica da aprendizagem. Porto Alegre: Artes Médicas, 1997.

HOLANDA, A. B. Dicionário Aurélio Eletrônico. Versão 5.0. São Paulo: Positivo Informática, 2004.

JAPIASSU, H.; MARCONDES, D. Dicionário básico de Filosofia. 3. ed. Rio de Janeiro: Jorge Zahar, 2001. 
LACLAU, E. Emancipación y diferencia. Buenos Aires: Ariel, 1996.

MARCELLINO, N. C. A dicotomia teoria/prática na Educação Física. Motrivivência, Florianópolis, v. 7, n. 8, p. 73-78, 1995. Disponible en:

https://periodicos.ufsc.br/index.php/motrivivencia/article/view/22598/20592. Acceso el: 25 jun. 2014.

MEZZAROBA, C. A formação de professores de Educação Física: tensões e possibilidades do professor-reflexivo e do professor-pesquisador. Corpoconsciência, Cuiabá, v. 20, n. 1, p. 109-123, 2016. Disponible en:

http://periodicoscientificos.ufmt.br/ojs/index.php/corpoconsciencia/article/download/4154/ 2930. Acceso el: 20 abr. 2020.

PINTO, L. M. S. M. Na prática a teoria é outra. Será mesmo?. Motrivivência, Florianópolis, v. 7, n. 8, p. 134-138, 1995. Disponible en:

https://periodicos.ufsc.br/index.php/motrivivencia/article/view/22604/20598. Acceso el: 25 jun. 2014.

POLIZEL, A. Percepções do movimento Escola sem Partido: currículos pastorais e o professor como catequista. Amazônida, Manaus, v. 4, n. 1, p. 1-16. 2019. Disponible en: http://www.periodicos.ufam.edu.br/amazonida/article/view/4942. Acceso el: 9 nov. 2019.

RAE - Real Academia Española. Diccionario de la lengua española. 23. ed. Madrid: RAE, 2019. Disponible en: https://dle.rae.es/. Acceso el: 5 mar. 2020.

RANCIÈRE, J. O mestre ignorante: cinco lições sobre a emancipação intelectual. 3. ed. Belo Horizonte: Autêntica, 2013.

SCHÖN, D. A. Formar professores como profissionais reflexivos. In: NÓVOA, A. Os professores e sua formação. Lisboa: Dom Quixote, 1992. p. 79-91.

SILVA, T. T. Teoria cultural e educação: um vocabulário crítico. Belo Horizonte: Autêntica, 2000.

SOUTHWELL, M. El análisis político del discurso y la cuestión de la politicidad de la educación. In: PADIERNA JIMÉNEZ, M. P.; GARCÍA CONTRERAS, M. (Org.). Lo político en las subjetivaciones: una década de investigaciones desde el análisis político de discurso. Investigación Social y Análisis Político de Discurso. Madrid: Plaza y Valdés, 2007.

STENHOUSE, L. An introduction to curriculum research and development. London: Heinemann, 1975.

STENHOUSE, L. Investigación y desarrollo del curriculum. Madrid: Morata, 1991.

TARDIF, M. Saberes profissionais dos professores e conhecimentos universitários: elementos para uma epistemologia da prática profissional dos professores e suas 
consequências em relação à formação para o magistério. Revista Brasileira de Educação, Belo Horizonte, n. 13, p. 5-24, 2000. Disponible en:

http://educa.fcc.org.br/pdf/rbedu/n13/n13a02.pdf. Acceso el: 20 abr. 2020.

Cristiano Mezzaroba, Universidad Federal de Sergipe (UFS), Departamento de Educación Física, Programa de Posgrado en Educación

iDhttp://orcid.org/0000-0003-4214-0629

Doctor en Educación, máster en Educación Física y licenciado en Educación Física y Ciencias Sociales por la Universidad Federal de Santa Catarina (UFSC).

Contribución de autoría: Participó en todas las etapas de la preparación y desarrollo del artículo.

Lattes: http://lattes.cnpq.br/1835801891069733

E-mail: cristiano_mezzaroba@yahoo.com.br

Nicolás Carriquiriborde, Universidad Nacional de La Plata (UNLP), Facultad de Humanidades y Ciencias de la Educación, Curso de Maestría en Educación

ii Dhttps://orcid.org/0000-0003-3656-7984

Profesor en Educación Fisica. Maestría de la Facultad de Humanidades y Ciencias de la Educación de la UNLP. Centro Interdisciplinario Cuerpo, Educación y Socidedad - Instituto de Investigaciones en Humanidad y Ciencias Sociales.

Contribución de autoría: Participó en todas las etapas de la preparación y desarrollo del artículo.

E-mail: nicolas.carriquiriborde@gmail.com

Editor responsable: Hugo Heredia Ponce

Pareceristas ad hoc: Marta Extremera y Manuel Romero Oliva

\section{Cómo citar este artículo (ABNT):}

MEZZAROBA, Cristiano; CARRIQUIRIBORDE, Nicolás. Teoría y práctica: cuestiones imprescindibles a la práctica educativa. Educ. Form., Fortaleza, v. 5, n. 3, p. 1-20, 2020. Disponible en: https://revistas.uece.br/index.php/redufor/article/view/2807.

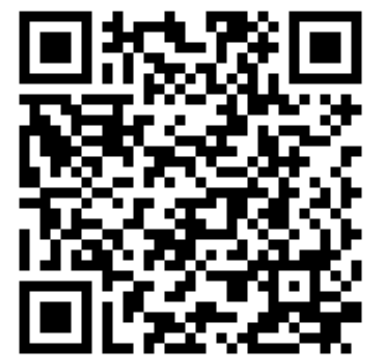

Recibido el 18 de febrero de 2020.

Aprobado el 15 de mayo de 2020.

Publicado el 27 de mayo de 2020. 